\title{
Partizipative Entscheidungsfindung (PEF) - Patient und Arzt als Team
}

\author{
Shared Decision Making (SDM) - Patient and Physician as a Team
}

Christiane Bieber, Kathrin Gschwendtner, Nicole Müller, Wolfgang Eich

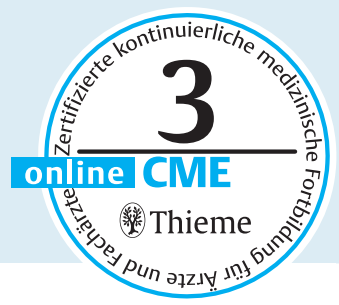

Klinik für Allgemeine Innere Medizin und Psychosomatik, Zentrum für Psychosoziale Medizin, Universitätsklinikum Heidelberg

\section{Zusammenfassung \\ $\nabla$}

Der Artikel gibt einen Überblick über das Konzept der Partizipativen Entscheidungsfindung (PEF), das von vielen Akteuren des Gesundheitssystems als besonders günstige Form der Arzt-Patient-Interaktion verstanden wird. Die PEF wird abgegrenzt gegen weitere Modelle der Arzt-PatientInteraktion wie das Paternalistische Modell und das Informationsmodell. Neben den Akzeptanzwerten von PEF in der Bevölkerung und unter Ärzten wird auch auf mögliche Barrieren in der Umsetzung eingegangen. Anwendungsbereiche für die PEF werden besprochen und Strategien sowie Hilfsmaterialien zur Umsetzung im individuellen Arzt-Patient-Kontakt dargestellt und anhand eines onkologischen Fallbeispiels vertieft. Effekte der PEF für Patienten und für Behandler werden aufgezeigt. Nach Eingehen auf wichtige Quellen von PEF erfolgt die Einordnung vor dem aktuellen gesundheitspolitischen Hintergrund in Deutschland.

\section{Abstract $\nabla$}

The article provides an overview on Shared Decision Making (SDM), which is considered as the ideal form of physician-patient-interaction by many stakeholders of the health care system. SDM is distinguished from other models of physician-patient-interaction such as the paternalistic model and the information model. Besides the degree of acceptance of SDM in the general population and among physicians, barriers for its implementation will be reported. Indications for SDM as well as strategies and support material for its use in individual consultations will be discussed and illustrated by an oncological case study. Effects of SDM for patients as well as for clinicians will be highlighted. After background information on origins of SDM, its significance with regard to health policy in Germany is discussed.

\section{Lernziele}

$\nabla$

Dieser Fortbildungsartikel...

- gibt einen Überblick über das Konzept der Partizipativen Entscheidungsfindung (PEF) sowie über weitere Modelle der Arzt-Patient-Interaktion

- erläutert die Umsetzung von PEF im Rahmen des individuellen Arzt-Patienten-Kontaktes

- erläutert Indikationen, Effekte und Argumente für den Einsatz von PEF

- erläutert den gesundheitspolitischen Hintergrund der Patientenbeteiligung in Deutschland

\section{Einführung}

Der Begriff „Partizipative Entscheidungsfindung“ - PEF (englisch auch: „Shared Decision Making“ - SDM) steht für eine partnerschaftliche Entscheidungsfindung im medizinischen Kontext, die aus einem patientenzentrierten Ansatz heraus erwächst.

Definiert wird Partizipative Entscheidungsfindung (PEF) als „ein Interaktionsprozess mit dem Ziel, unter gleichberechtigter aktiver Beteiligung von Patient und Arzt auf Basis geteilter Information zu einer gemeinsam verantworteten Übereinkunft zu kommen." [1]

Die Partizipative Entscheidungsfindung wird mittlerweile von vielen Akteuren des Gesundheitssystems als besonders günstige Form der Arzt-Patient-Interaktion verstanden [2], da sie den mündigen, informierten Patienten in den Mittelpunkt des Handelns stellt. Patient und Arzt begegnen sich in diesem Modell als gleichberechtigte Partner. Im PEF-Modell finden sowohl mögliche Autonomiewünsche des Patienten als auch
VNR

2760512016149753810

Schlüsselwörter

- Partizipative Entscheidungsfindung (PEF)

- Arzt-Patient-Interaktion

- Medizinische Entscheidungshilfe

- Patientenzentrierung

Keywords

- Shared Decision Making (SDM)

- physician-patient-interaction

- Patient Decision Aid (PtDA)

- patient-centeredness
Bibliografie

Dol http://dx.doi.org/ 10.1055/s-0042-105277 Psychother Psych Med 2016; 66: 195-207

(c) Georg Thieme Verlag KG Stuttgart · New York ISSN 0937-2032

\section{Korrespondenzadresse}

PD Dr. med. Christiane Bieber Klinik für Allgemeine Innere Medizin und Psychosomatik, Zentrum für Psychosoziale Medizin, Universitätsklinikum Heidelberg

Thibautstraße 2

69115 Heidelberg

Christiane.Bieber@med.uni-

heidelberg.de 


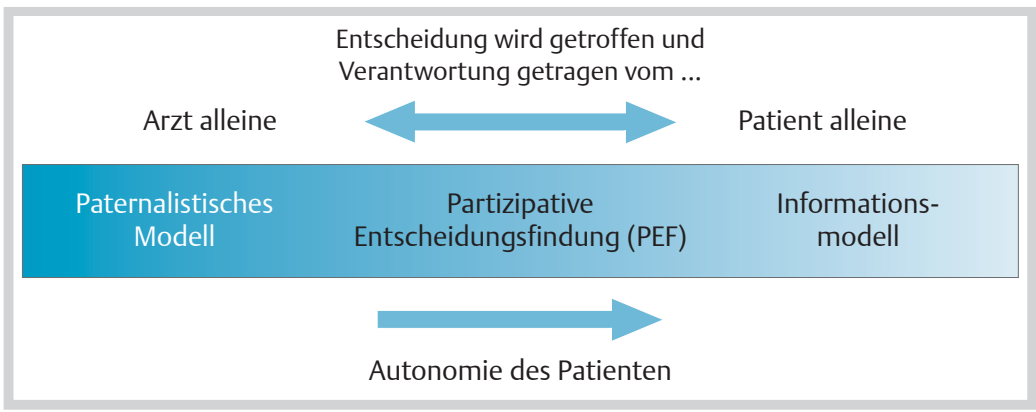

Abb.1 Das Spektrum der Arzt-Patient-Interaktion [7].

sein Bedarf nach Anlehnung und Anleitung durch den Arzt individuelle Berücksichtigung.

Als Voraussetzung für eine Partizipative Entscheidungsfindung in der Medizin gilt eine ethische Grundhaltung der Behandler, die ein Selbstbestimmungsrecht des Patienten als Wert an sich akzeptiert [3] und eine Patientenzentrierung anstrebt.

PEF ist bei wichtigen Behandlungsentscheidungen relevant, aber auch andere Entscheidungen können partizipativ getroffen werden (z.B. Durchführung diagnostischer Tests oder Screeningmaßnahmen, Krankschreibung, Ort der Behandlung, Beendigung der Behandlung).

Während eine patientenzentrierte Herangehensweise in den psychosozialen Fächern der Medizin heute eher als Selbstverständlichkeit gilt, ist sie im somatischen Bereich der Medizin noch nicht flächendeckend angekommen. Insofern kann das PEF-Konzept auch als ein Vehikel begriffen werden, die Verbreitung einer patientenzentrierten Haltung in der somatischen Medizin zu fördern.

\section{Der Bedarf für PEF}

\section{$\nabla$}

Wichtige Impulse hin zu einer Stärkung der Autonomie des Patienten ergeben sich einerseits aus der Tatsache, dass evidenzbasierte Therapieoptionen in den letzten Jahren durch den wissenschaftlichen Fortschritt in vielen Bereichen der Medizin rasant zunehmen. Dadurch bestehen zunehmend auch Wahlmöglichkeiten zwischen verschiedenen Behandlungsoptionen, die sich hinsichtlich Ergebnis, Risiken und Nutzen stark unterscheiden können. Relevant wird dies ganz besonders bei schwerwiegenden, potenziell lebensbedrohlichen Erkrankungen sowie bei chronischlebensbegleitenden Erkrankungen. Die Perspektive des Patienten, seine Vorstellungen und Werte, seine Risikobereitschaft, sein Sicherheitsbedürfnis sollten daher schon aus einer ethischen Haltung heraus Berücksichtigung finden. Andererseits können sich Patienten mittlerweile umfassender informieren, da Gesundheitsinformationen z.B. über das Internet zunehmend auch für medizinische Laien verfügbar sind. Das traditio- nelle Rollenverständnis zwischen Ärzten und ihren Patienten wird damit infrage gestellt und Behandler treffen zunehmend auf Patienten, die gut informiert sind und sich aktiv an Behandlungsentscheidungen beteiligen wollen.

\section{Charakterisierung der PEF}

$\nabla$

Das Konzept der PEF fand zunächst im angloamerikanischen Sprachraum die größte Beachtung und wurde seit den 90er-Jahren in einigen konzeptionellen Veröffentlichungen dargelegt [4-7]. Die Kanadierin Cathy Charles [5] benannte in diesem Zusammenhang erstmals die vier Charakteristika der PEF:

- Mindestens zwei Personen, der Arzt und der Patient, sind in den Entscheidungsprozess bzgl. einer Behandlung involviert

- Sowohl der Arzt als auch der Patient teilen ihre Informationen miteinander

- Arzt und Patient nehmen an dem Entscheidungsprozess teil, indem sie sich gegenseitig ihre Behandlungspräferenzen offen legen

- Arzt und Patient treffen eine Behandlungsentscheidung und stimmen darin überein, diese Behandlung durchzuführen

Um die Besonderheiten des PEF-Modells würdigen zu können, ist es notwendig, es gegen andere mögliche Formen der Arzt-Patient-Interaktion abzugrenzen, was im folgenden Abschnitt erfolgt.

\section{Das Spektrum der Arzt-Patient-Inter- aktion}

Innerhalb des Gesundheitswesens können mindestens drei unterschiedliche Ausgestaltungsweisen der Arzt-Patient-Interaktion zur Anwendung kommen, die jeweils mit einem variierenden Rollenverständnis von Arzt und Patient einhergehen. Die Partizipative Entscheidungsfindung nimmt im Spektrum dieser möglichen Formen hinsichtlich der vorhandenen Patientenautonomie eine Mittelstellung zwischen dem Paternalistischen Modell und dem Informationsmodell ein (vgl. auch [6-8]) ( $\bullet$ Abb.1).

In der Praxis finden sich dabei auch Mischformen, aus didaktischen Gründen sollen hier allerdings die Idealtypen beschrieben werden.

Beim Modell der Partizipativen Entscheidungsfindung treffen Arzt und Patient gemeinsam die Entscheidung, nachdem sie medizinische und auch behandlungsrelevante persönliche Informationen ausgetauscht und ihre Rollen- und Behandlungspräferenzen offengelegt haben. Die Verantwortung für die Umsetzung der Behandlung wird somit geteilt [5].

Beim Paternalistischen Modell in seiner reinsten Form beschränkt sich die Patientenautonomie auf ein Minimum [5]. Die medizinischen Informationen sind nur dem Arzt bekannt, welcher nach- 


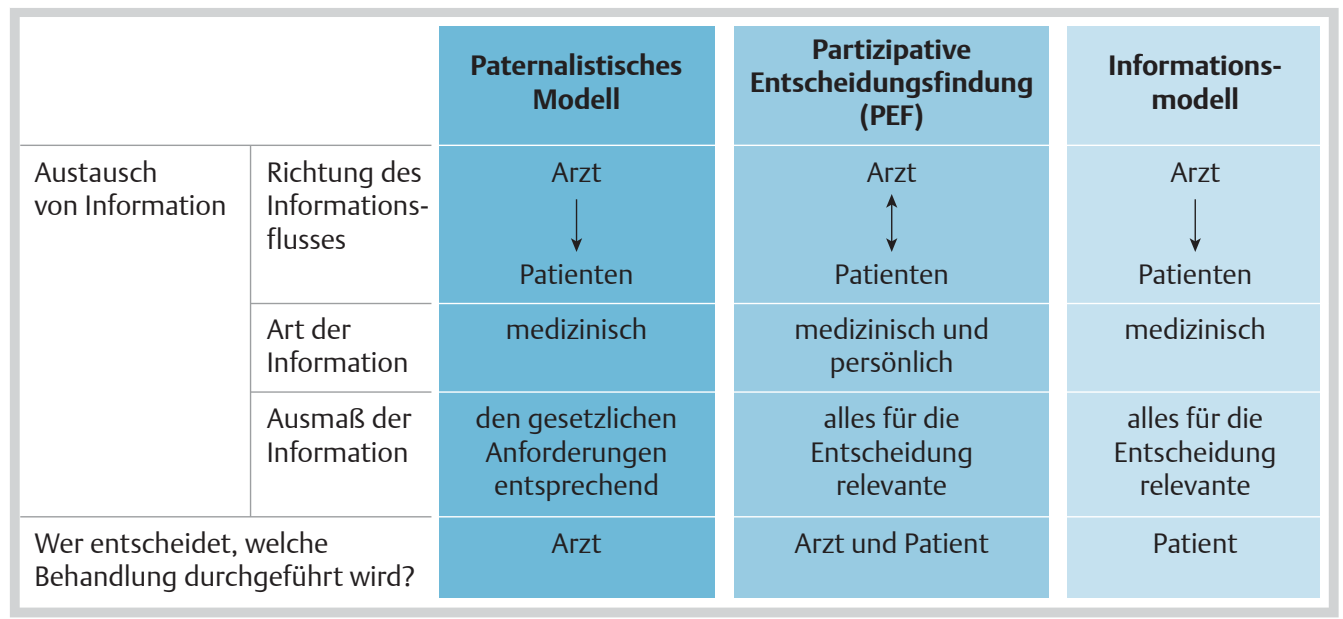

Abb.2 Die Bedeutung der Information in den Modellen der medizinischen Entscheidungsfindung [4].

folgend alleinverantwortlich, im vermeintlich besten Interesse des Patienten entscheidet, ohne allerdings dessen explizite Bedürfnisse zu kennen. Neben Elementen der Fürsorge und der wohlwollenden Beratung birgt dieses Modell die Gefahr der Bevormundung und partiellen Entmündigung des Patienten. Das Paternalistische Modell wird traditionell in der Medizin angewandt, herrscht im deutschen Medizinsystem auch heute noch vor, befindet sich jedoch mittlerweile aus verschiedensten Gründen auf dem Rückzug.

Am diametralen Pol dieses Spektrums befindet sich das Informationsmodell, bei welchem die Patientenautonomie ein Maximum erreicht [5]. Der Arzt stellt seinem Patienten alle relevanten medizinischen Informationen zur Verfügung, nimmt sich aus dem Prozess der Entscheidungsfindung jedoch heraus. Der Patient wägt die erhaltenen Informationen vor dem Hintergrund seiner eigenen Befürchtungen und Erwartungen ab und entscheidet alleinverantwortlich. Das Informationsmodell knüpft an das Kundenmodell der freien Wirtschaft an und legt damit eine andere Beziehungs- und Hierarchiestruktur zugrunde [8]. Der Arzt fungiert als Experte und Dienstleister, der Patient befindet sich in der Rolle des Kunden. Eine mögliche Gefahr liegt bei diesem Modell in der Überforderung des Patienten.

Während beim Paternalistischen Modell sowie beim Informationsmodell die ausgetauschten Informationen hauptsächlich medizinischer Natur sind, werden im PEF-Modell auch behandlungsrelevante persönliche Informationen zwischen Arzt und Patient ausgetauscht [9] ( $\bullet$ Abb.2). Zwei Personen treffen hier gleichberechtigt aufeinander, der Arzt ist Experte für das medizinische Fachwissen, dem Patienten sind seine eigenen persönlichen Werte, Wünsche und Vorstellungen bekannt. Erwartungen, aber auch Sorgen, Ängste und Befürchtungen des Patienten dürfen und sollen explizit thematisiert werden. Auch der Arzt darf seine persönlichen Behandlungserfah- rungen einbringen. Es obliegt dabei dem Arzt, durch Herstellen einer vertrauensvollen Atmosphäre und ggf. konkretes Nachfragen, den Austausch über diese Themen überhaupt erst zu ermöglichen [7].

\section{Akzeptanz der PEF \\ $\nabla$}

Seit Beginn entsprechender Erhebungen Ende des 20. bzw. Anfang des 21. Jahrhunderts ist anhand großer Datensätze gut belegt, dass sich die Mehrheit der Bürger in Deutschland $[10,11]$ und auch in anderen europäischen Ländern [12] eine partnerschaftliche Beteiligung bei medizinischen Entscheidungen wünscht. Beispielsweise sprechen sich in den seit 2001 - 2012 jährlich durchgeführten Erhebungen des Gesundheitsmonitors der Bertelsmannstiftung relativ konstant etwas mehr als die Hälfte aller Befragten für PEF als ihr Wunschmodell aus ( Abb.3).

Während die theoretische Zustimmung zu PEF bei Ärzten sogar noch stärker auszufallen scheint als bei den Bürgern und sich in einer Umfrage unter 500 Ärzten sogar 75\% für die Partizipative Entscheidungsfindung als ihr Wunschmodell im Umgang mit Patienten aussprechen [13], spiegelt sich dies in der Behandlungsrealität offensichtlich nicht wider. Anspruch und Wirklichkeit klaffen weit auseinander ( $\bullet$ Abb.4): Patienten haben z.B. im Vergleich zu den behandelnden Ärzten deutlich seltener den Eindruck, dass ihre Vorstellungen über die Behandlung miteinbezogen werden (37\% der Patienten vs. 66\% der Ärzte), oder ihnen Vorund Nachteile verschiedener Behandlungen erklärt wurden (51\% der Patienten vs. 89\% der Ärzte).

Studien zeigten überdies, dass Ärzte ihre eigenen kommunikativen Fertigkeiten über- und den Kommunikationsbedarf ihrer Patienten unterschätzen [14].

Aus ärztlicher Sicht sind die wichtigsten Barrieren für die Umsetzung von PEF im klinischen Alltag

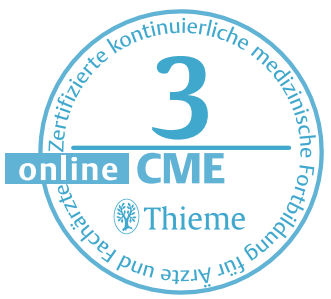




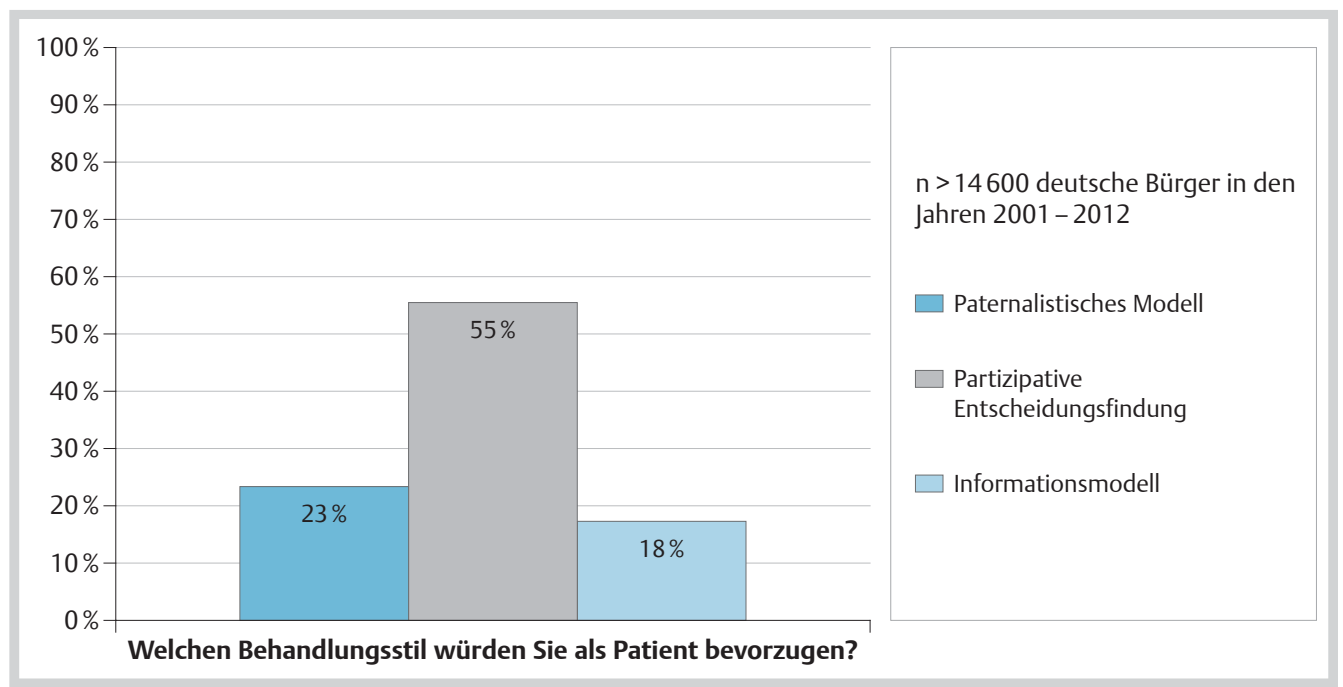

Abb.3 Bevorzugtes Modell der Entscheidungsfindung in Deutschland [11].

ein wahrgenommener Zeitdruck sowie die Annahme, dass ein Patient aufgrund seiner Charakteristika oder aufgrund der klinischen Situation nicht für den PEF-Ansatz geeignet sei [15]. Es gibt jedoch Hinweise, dass selbst Patienten, die eine eher passive Rolle im Entscheidungsfindungsprozess wünschen, von einer aktiven Einbeziehung besonders profitieren, weil die passive Haltung möglicherweise eher durch einen Mangel an Selbstwirksamkeitserwartungen als durch ein echtes Desinteresse an Beteiligung zu verstehen ist [16]. Auch scheint PEF in ärztlichen Konsultationen nicht notwendigerweise den Zeitaufwand zu erhöhen [16].

In einer repräsentativen Übersichtsarbeit zeigte sich, dass Patienten die Hauptbarriere für eine aktive Entscheidungsteilhabe in einem Wissens- sowie einem Machtungleichgewicht zwischen sich und den Ärzten sehen. Es wurde deutlich, dass es nicht so sehr am Willen der Patienten zur Beteiligung liegt, sondern vielmehr an den durch die behandelnden Ärzte eingeräumten Möglichkeiten und Gelegenheiten zur Partizipation [17].

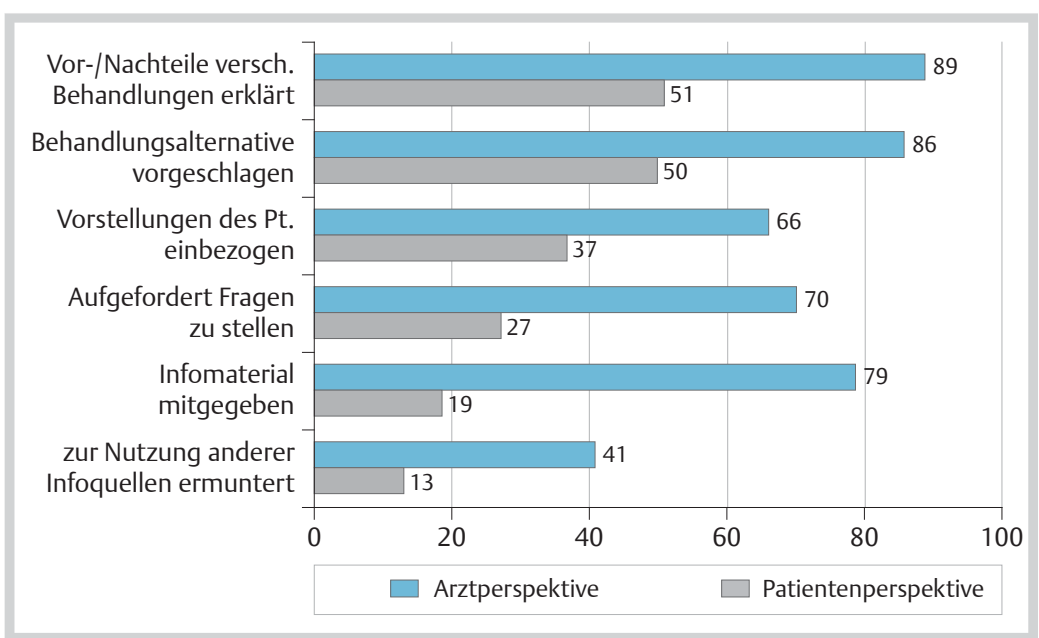

Abb.4 Diskrepante Perspektiven: Verhalten des Hausarztes aus Arzt- und aus Patientensicht ( $n=502$ Ärzte und $n=1512$ Bürger) [14].

\section{Indikationen für PEF}

$\nabla$

In der Literatur werden zahlreiche Faktoren benannt, die die Anwendung des PEF-Modells als indiziert erscheinen lassen $[4,9,18]$ :

Eine Entscheidungsfindung im Einklang mit PEFPrinzipien sollte insbesondere dann erwogen werden, wenn verschiedene evidenzbasierte Behandlungsmethoden zur Wahl stehen, die als gleichwertig erachtet werden können, die sich aber hinsichtlich ihrer Konsequenzen für das weitere Leben des Patienten unterscheiden.

Dabei sollte bedacht werden, dass Behandlungsoptionen sich in ihrem Risiko-Nutzen-Profil unterscheiden können, unterschiedliche Konsequenzen für das körperliche und psychische Wohlbefinden des Patienten mit sich bringen können und das Ergebnis der Behandlung oft ungewiss ist. Dies trifft sowohl bei schwerwiegenden medizinischen Entscheidungen (z.B. in der Onkologie) als auch bei der Behandlung chronischer Erkrankungen zu. Weitere Indikationen für die Anwendung des PEF-Modells können der Wunsch des Patienten nach Beteiligung an der Entscheidungsfindung sein, aber auch, dass der Arzt die Verantwortung nicht alleine übernehmen kann und will.

Whitney [19] entwickelte in diesem Kontext ein Modell zur Auswahl der Herangehensweise an medizinische Entscheidungen, das sowohl den Grad an medizinischer Gewissheit/Sicherheit als auch den Grad der Bedeutsamkeit für den Patienten berücksichtigt ( $\bullet$ Abb. 5). Das Modell soll Ärzten bei der Einschätzung behilflich sein, welche Herangehensweise an die medizinische Entscheidungsfindung am ehesten indiziert ist. Bei diesem Modell liegt ein sehr breiter Anwendungsbereich für PEF vor, Arzt und Patient können demnach oft gemeinsam entscheiden. 
Ist die Bedeutsamkeit der medizinischen Maßnahme für den Patienten gering und gibt es aus medizinischer Sicht ohnehin nur eine mögliche Vorgehensweise, ist die Gewissheit bezüglich des korrekten Vorgehens demnach groß und der Arzt eher dazu geneigt, die Führung zu übernehmen und paternalistisch vorzugehen (siehe rechte untere Ecke im Modell nach Whitney). Als Beispiel hierfür kann die Indikation zur Appendektomie bei einer akuten Appendizitis gesehen werden. Auch in Notfallsituationen oder in emotionalen bzw. kognitiven Überforderungssituationen des Patienten ist in der Regel ein paternalistisches Vorgehen sinnvoll. Anders verhält es sich in Situationen, in denen die Bedeutsamkeit der Maßnahme für den Patienten sehr groß ist, die Gewissheit über die beste evidenzbasierte Vorgehensweise aber gering, weil es entweder sehr viele evidenzbasierte Optionen gibt, oder aber keine der vorhandenen Optionen als evidenzbasiert gelten kann. In diesen Fällen kann die Entscheidung hauptsächlich durch den Patienten getroffen werden (siehe linke obere Ecke im Modell). Ein Beispiel hierfür kann die Entscheidung zu einem kosmetischen Eingriff sein. Ein potenzieller Konflikt zwischen Patient und Arzt ist vorprogrammiert, wenn die Bedeutsamkeit für den Patienten sehr groß ist und die medizinische Evidenz eindeutig für eine bestimmte Vorgehensweise spricht, die der Präferenz des Patienten aber widerspricht (siehe rechte obere Ecke im Modell). Ein Beispiel hierfür kann eine schwere Anämie mit der Notwendigkeit einer Bluttransfusion sein, die der Patient jedoch aus z.B. religiösen Gründen ablehnt. Wie nun die Umsetzung der PEF im individuellen Arzt-Patient-Kontakt erfolgen kann, wird im folgenden Abschnitt erläutert.

\section{Die Umsetzung von PEF im individuellen Arzt-Patient-Kontakt \\ $\nabla$}

Eine Voraussetzung für PEF ist eine patientenzentrierte Grundhaltung des Arztes, sowie eine Offenheit für die Bedürfnisse des Patienten. PEF kann nur in einer vertrauensvollen, partnerschaftlichen Atmosphäre gelingen [18].

Hervorzuheben ist hierbei, dass zwar die Patientenautonomie respektiert wird, vonseiten des Arztes eine Teilhabe an der Therapieentscheidung aber nicht erzwungen werden kann. Patienten sollten in dem von ihnen bevorzugten Ausmaß beteiligt werden [20]. Diese Herangehensweise erfordert ein gewisses Maß an Flexibilität seitens des Arztes, sich auf die jeweiligen Beteiligungsbedürfnisse des Patienten einzustellen und den eigenen Konsultationsstil entsprechend anzupassen, denn auch ein Erzwingen von Patientenbeteiligung kann eine Form von bevormun-

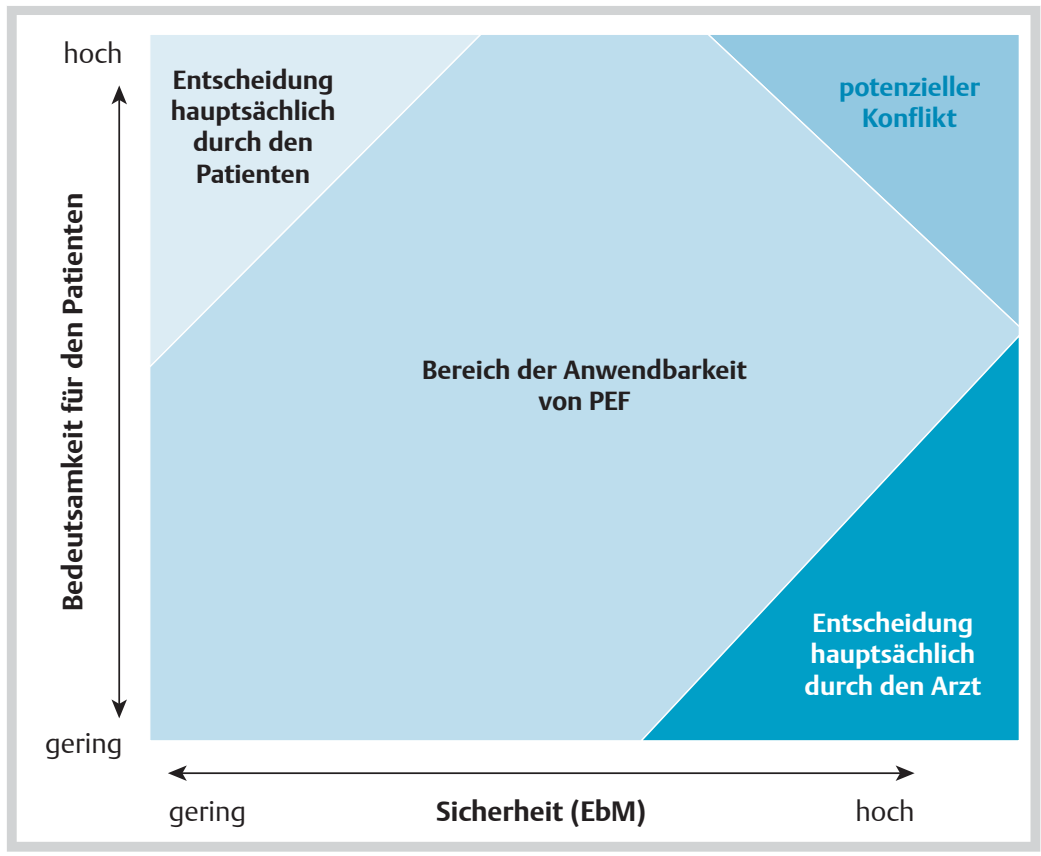

Abb.5 Modell zur Anwendbarkeit von PEF [18].

dendem Paternalismus darstellen [18] und sollte daher unterbleiben.

Im individuellen Arzt-Patient-Kontakt kann PEF gelingen, wenn bestimmte Umsetzungsschritte Berücksichtigung finden, die von Towle et al. [6] und von Elwyn et al. [18] herausgearbeitet wurden. Im Wesentlichen können diese Umsetzungsschritte unter 6 Punkten zusammengefasst werden, die nicht in starrer Reihenfolge durchlaufen werden müssen, sich aber sinnvollerweise in der Konsultation wiederfinden sollten, um PEF zu ermöglichen ( $\bullet$ Abb.6).

Die Prozessschritte helfen dabei, das Konsultationsgespräch in 3 Phasen zu gliedern. In der Anfangsphase („Team Talk“) sollte seitens des Arztes transparent gemacht werden, dass eine Entscheidung ansteht und mehrere Möglichkeiten bestehen, mit dem Problem umzugehen. Gleichzeitig sollte das Angebot für ein partnerschaftliches Rollenverständnis erfolgen. Die Informationsphase beinhaltet einen Austausch von fachlichen und persönlichen Informationen („Option Talk“). Es sollte vom Arzt erfasst werden, ob die Informationen verstanden wurden, wie die Sichtweise des Patienten ist und welcher Informations- und Beratungsbedarf noch besteht. In der Abschlussphase („Decision Talk“) sollte geklärt werden, welche Behandlungs- und Rollenpräferenzen auf beiden Seiten bestehen und es sollten Vereinbarungen getroffen oder auch vertagt werden.

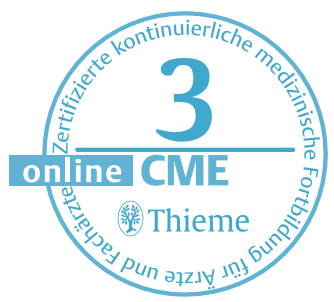




$\left.\begin{array}{l|l}\begin{array}{l}\text { 1. Problemdefinition } \\ \text { Mitteilen, dass eine Entscheidung ansteht } \\ \text { 2. Gleichwertigkeit der Behandlungsoptionen und der Partner } \\ \text { Es gibt mehr als einen Weg, um mit dem Problem umzugehen }\end{array} & \begin{array}{l}\text { Anfangsphase } \\ \text { "Team Talk“ }\end{array} \\ \begin{array}{l}\text { 3. Behandlungsmöglichkeiten beschreiben } \\ \text { Über Optionen und deren Vor- und Nachteile informieren }\end{array} \\ \begin{array}{l}\text { 4. Verständnis, Gedanken, Erwartungen und } \\ \text { Befürchtungen erfragen } \\ \text { Die Sicht des Patienten mit einbeziehen }\end{array} \\ \begin{array}{l}\text { 5. Rollenpräferenz und Behandlungspräferenz klären } \\ \text { und Entscheidungsfindung } \\ \text { Beteiligungswunsch ermitteln und Entscheidung herbeiführen } \\ \text { 6. Vereinbarungen treffen } \\ \text { Vereinbarungen zur Umsetzung der Entscheidung treffen }\end{array}\end{array}\right\} \begin{aligned} & \text { Informations- } \\ & \text { phase } \\ & \text { "Option Talk“ }\end{aligned}$

Abb.6 Prozessschritte zur Umsetzung von $\operatorname{PEF}[6,17,20]$.

\section{Strategische Ansätze und Hilfsmaterialien zur Umsetzung von PEF \\ $\nabla$}

Um die Umsetzung von PEF im individuellen ArztPatienten-Kontakt zu fördern, kann sowohl auf Arzt- als auch auf Patientenseite angesetzt werden.

Viele Ärzte befürworten zwar ein Vorgehen nach PEF-Prinzipien, die Umsetzung scheitert dann jedoch häufig an mangelnden Kommunikationsfertigkeiten [18]. Die hierfür notwendigen Kompetenzen gehen über die grundlegenden Kommunikationsfertigkeiten hinaus, die bislang standardmäßig in der medizinischen Ausbildung vermittelt wurden [22].

Ärzte haben jedoch die Möglichkeit, in speziellen PEF-Kommunikationstrainings zu lernen, Patienten bei Behandlungsentscheidungen mit einzubeziehen [23-26]. Ein wichtiges Element von PEFSchulungen sind Elemente der Risikokommunikation, da sowohl Patienten als auch ihre Ärzte sich mit dem Verständnis von Statistiken schwer tun und diese leicht fehlinterpretieren [27].

Auf Patientenseite gibt es ebenfalls Ansatzmöglichkeiten, die Umsetzung von PEF zu erleichtern. Während Patientenschulungen einen möglichen, aber bislang eher selten beschrittenen Weg darstellen [28], sind sogenannte medizinische Entscheidungshilfen für Patienten (Patient Decision Aids- PtDAs [29]) eine insbesondere in Nordamerika und Großbritannien verbreitete und gelegentlich bereits in der Routineversorgung eingesetzte Variante.

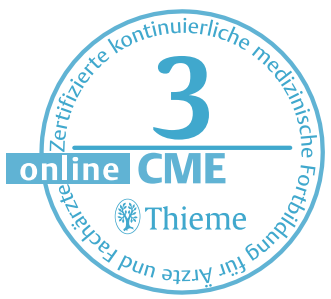

Entscheidungshilfen können in unterschiedlicher Form vorliegen, wie z.B. als Broschüre, Video oder Computerprogramm. Sie informieren den Patienten über vorhandene Behandlungsoptionen und Outcomes bei einer umschriebenen Fragestellung und stellen systematisch Vor- und Nachteile der Optionen sowie Risiken und Nutzen gegenüber. Meist enthalten sie auch Elemente, die zu einer Klärung persönlicher Werte und Präferenzen beitragen und den Patienten somit beim Gewichten und Abwägen der verschiedenen Behandlungsoptionen unterstützen. Medizinische Entscheidungshilfen sollten international anerkannten Qualitätsstandards (IPDAS-Kriterien) [30]) genügen. In Kanada aktualisiert z.B. das an die Universität in Ottawa angegliederte OHRI (Ottawa Hospital Research Institute) regelmäßig ein Verzeichnis mit über 100 englischsprachigen Entscheidungshilfen, die größtenteils online verfügbar sind (www.ohri.ca/decisionaid). Weitere Organisationen in Nordamerika, z. B. FINDIM (Foundation for Informed Medical Decision Making) oder „Health Dialogue“ sind im großen Stil mit der Erstellung und dem Vertrieb von Entscheidungshilfen beschäftigt.

Onkologisch tätigen Ärzten bietet die englischsprachige Internetplattform „Adjuvant! Online“ (www.adjuvantonline.com) die Möglichkeit, für Patienten mit Krebserkrankungen in frühen Stadien (z.B. Brustkrebs und Darmkrebs) anhand von Online-Tools sehr individuelle Berechnungen hinsichtlich der Prognose mit und ohne diverse adjuvante Therapiemaßnahmen vorzunehmen. Diese Berechnungen werden grafisch vom System direkt und gut verständlich visualisiert und können so in Konsultationen mit Patienten unterstützend als Entscheidungshilfe eingesetzt werden.

In Deutschland hingegen werden medizinische Entscheidungshilfen bisher eher selten in der Routinebehandlung eingesetzt. Das Interesse an medizinischen Entscheidungshilfen nimmt erst in den letzten Jahren zu. Teilweise engagieren sich Krankenkassen bei der Entwicklung und Verbreitung medizinischer Entscheidungshilfen (z.B. AOK: Entscheidungshilfe Brustkrebs [31]).

Aktuell befindet sich unter Federführung von Prof. Dr. Donner-Banzhoff an der Universität in Marburg (in Kooperation mit den Universitäten Düsseldorf und Rostock) eine Online-Bibliothek mit deutschsprachigen Entscheidungshilfen zu wichtigen allgemeinmedizinischen Fragestellungen im Aufbau (www.arriba-hausarzt.de/arribalib). Mit einem bereits frei verfügbaren Onlineprogramm können Hausärzte für ihre Patienten unter Berücksichtigung individueller Komorbiditäten und Risikofaktoren das individuelle Risiko für einen Herzinfarkt oder einen Schlaganfall berechnen. In patientenfreundlicher grafischer Aufbereitung werden dabei die Effekte von Verhaltensänderungen oder medikamentösen Maßnahmen im individuellen Fall leicht verständlich dargestellt. Dieses persönliche Risikoprofil erleich- 
tert die Einbeziehung des Patienten bei der Abwägung z.B. für oder gegen eine Verhaltensänderung oder eine medikamentöse Behandlung. Es existieren weitere Beratungsmaterialien für Entscheidungssituationen bei Depression, KHK, Diabetes mellitus, orale Antikoagulation und PSAScreening.

\section{Wissenschaftlich belegbare Effekte von PEF}

Internationale Studien belegen günstige psychosoziale Effekte der Partizipativen Entscheidungsfindung $[29,32,33]$.

PEF-Interventionen (Entscheidungshilfen und/oder Ärztetrainings und Patiententrainings) führen zu einer Verbesserung der Arzt-Patient-Kommunikation und zu einer aktiveren Beteiligung von Patienten am medizinischen Entscheidungsprozess. Es kommt zu einer Zunahme des Wissens, einer besseren Risikowahrnehmung, zu realistischeren Erwartungen über die Behandlungsverläufe und zu geringeren Entscheidungskonflikten [32].

Die Ängstlichkeit der Patienten steigt durch die PEF-Interventionen nicht an, die Lebensqualität wird ebenfalls nicht beeinflusst. Hinsichtlich der Inanspruchnahme medizinischer Leistungen ist die Datenlage uneinheitlich. Weiterer Forschungsbedarf besteht hinsichtlich des Einflusses von PEF-Maßnahmen auf die Therapietreue, klinische Behandlungseffekte und gesundheitsökonomische Messgrößen [29, 32].

Auch Behandler profitieren von PEF mit ihren Patienten, da die Arzt-Patient-Beziehung gestärkt wird, sie Verantwortung abgeben können, ihre Zufriedenheit mit dem Patientenkontakt wächst und Folgekontakte ggf. kürzer ausfallen.

\section{Ein Anwendungsbeispiel für PEF aus der Praxis \\ $\nabla$}

Der 60-jährige Herr K. war bislang immer gesund. Er arbeitet als Gymnasiallehrer, ist glücklich verheiratet und hat 3 erwachsene Töchter sowie 2 Enkel. Das Ehepaar ist sozial gut eingebunden und Herr K. geht gemeinsam mit seiner Ehefrau aktiv einigen Hobbys nach. Bei einer Vorsorgeuntersuchung, zu der er sich als gesundheitsbewusster Mensch rein aufgrund seines Alters entschlossen hat, wird im Rahmen einer Koloskopie ein Kolonkarzinom diagnostiziert. Herr K. wird zügig operiert und es ist eine restlose Entfernung des Tumors (R0-Resektion) möglich. Die Tumorerkrankung von Herrn K. befindet sich noch in einem relativ frühen Stadium (Stadium II mit Risikofaktoren, T4 N0 M0 G2), allerdings liegt wegen ausgeprägter Tumorgröße ein Risikofaktor vor.
Gemäß Leitlinien zur Behandlung des Kolonkarzinoms ist in diesem Fall eine adjuvante Chemotherapie möglich, aber nicht zwingend erforderlich, denn Nutzen und Risiken der Chemotherapie halten sich in diesem speziellen Stadium aus medizinischer Sicht die Waage. Die aktuellen Leitlinien empfehlen daher, dass die persönlichen Präferenzen des Patienten bei dieser Therapieentscheidung miteinbezogen werden sollen. Herr K. sieht sich nun plötzlich mit der Frage konfrontiert: Chemotherapie ja oder nein? Wie soll Herr K. sich entscheiden? Er möchte diese Entscheidung im Sinne der Partizipativen Entscheidungsfindung gemeinsam mit seinem behandelnden Onkologen treffen. Auch seine Ehefrau bringt er zu dem Gespräch mit. Sein Onkologe setzt eine medizinische Entscheidungshilfe ein. In der Entscheidungshilfe sind zunächst die möglichen Behandlungspfade dargestellt, die für Herrn K. infrage kommen. Der Onkologe erläutert Herrn K. und dessen Ehefrau anhand eines Flussdiagramms die verschiedenen Optionen ( $\bullet$ Abb.7).

Herr K. kann entweder auf eine Chemotherapie verzichten und wird direkt in die Nachsorge eingeschlossen oder er entscheidet sich für eine Chemotherapie, wobei dann 3 verschiedene Chemotherapieprotokolle in Betracht gezogen werden können. Herr K. möchte von seinem Onkologen wissen, welchen Unterschied die Chemotherapie für seine Prognose machen wird und bekommt in diesem Zusammenhang folgende Risikotafeln gezeigt $(\bullet$ Abb.8):

Der Onkologe erläutert ihm anhand der Risikotafeln, wie das Rückfallrisiko innerhalb der nächsten 5 Jahre für Patienten in vergleichbarer Situation einzuschätzen ist. Herr K. erfährt, dass seine Prognose auch ohne Chemotherapie bereits recht gut ist. Denn ohne Chemotherapie werden 64 von 100 Patienten in ähnlicher Situation nach 5 Jahren weiterhin tumorfrei sein. Bei 29 von $100 \mathrm{~Pa}-$ tienten wird es allerdings zu einem Tumorrezidiv kommen und 7 von 100 Patienten werden innerhalb der nächsten 5 Jahre aus anderen Gründen verstorben sein. Mit einer adjuvanten Chemotherapie werden hingegen 10 weitere Patienten, also insgesamt 74 Patienten, nach 5 Jahren tumorfrei sein, und es wird bei 19 Patienten zu einem Tumorrezidiv gekommen sein. Alle 100 dieser Patienten können jedoch potenziell auch unerwünschte Wirkungen der Chemotherapie erfahren, wobei 64 dann überbehandelt gewesen wären, da sie ohnehin kein Rezidiv bekommen hätten. Die 19 mit Tumorrezidiv hätten die unerwünschten Wirkungen der Chemotherapie in Kauf genommen, um dann trotzdem wieder zu erkranken. Der Onkologe teilt Herrn K. auch mit, dass es sich hierbei um eine Statistik handelt und niemand ihm vorhersagen kann, welcher dieser Gruppen er nach 5 Jahren zuzuordnen sein wird. Herr K. hat jetzt verstanden, dass seine Heilungschancen bereits ohne Chemotherapie relativ gut sind, eine Chemotherapie die Heilungschancen

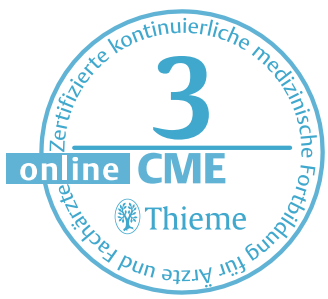




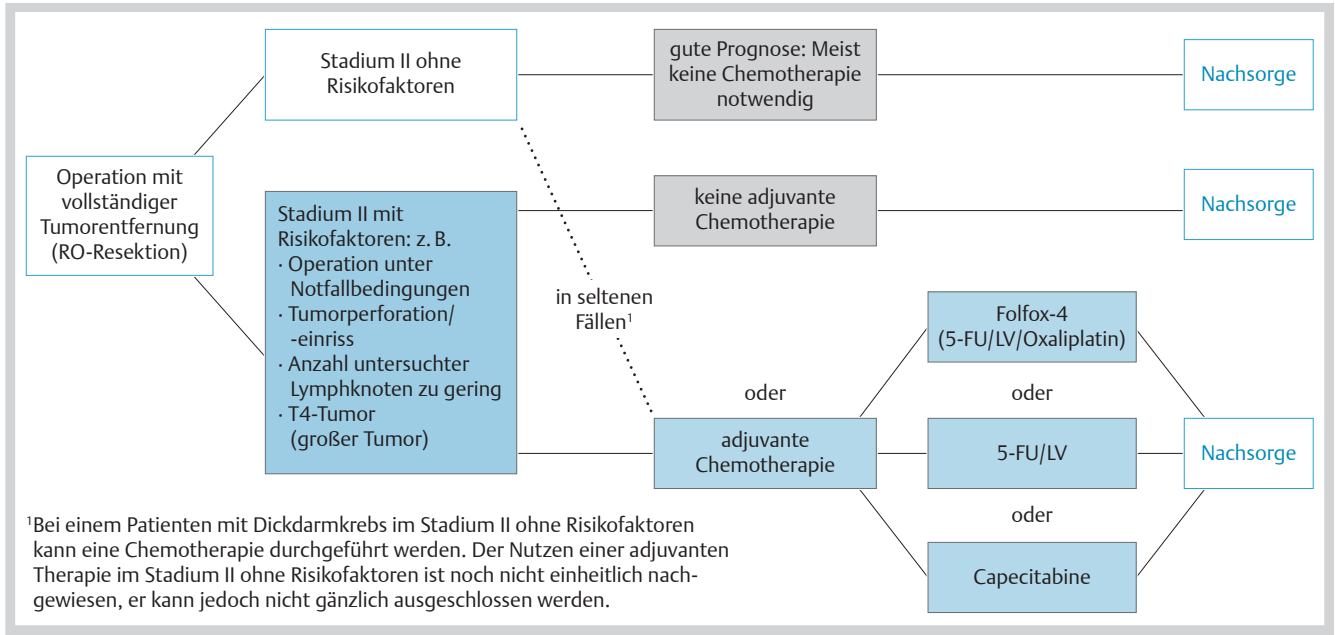

Abb.7 Übersicht über die Behandlungsmöglichkeiten bei Dickdarmkrebs im Stadium II [33].

29 von 100 Pat. erkranken erneut an Darmkrebs

Abb. 8 Das Risiko für einen Tumorrückfall bei Dickdarmkrebs im Stadium II mit Risikofaktoren - Situation ohne und mit Chemotherapie im Vergleich [33].

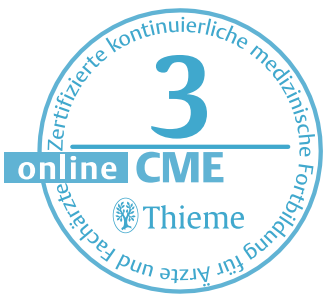

noch etwas verbessert, die Chemotherapie unerwünschte Wirkungen mit sich bringen kann und dass er trotz Chemotherapie einen Rückfall bekommen könnte. Dennoch tendiert er bei großem Sicherheitsbedürfnis, auch seiner Familie zuliebe, zu einer Chemotherapie. Sein Onkologe sieht dies ebenso. Gemeinsam entscheiden sich Herr K., seine Ehefrau und sein Onkologe daher zur Durchführung einer adjuvanten Chemotherapie.

Bei der Wahl des am besten für Herrn K. geeigneten Chemotherapeutikums setzt der Onkologe eine weitere Entscheidungstafel ein, um den Überblick zu erleichtern. Die drei zur Verfügung stehenden Chemotherapeutika unterscheiden sich nur leicht hinsichtlich ihrer Effektivität, jedoch deutlich hinsichtlich der Applikationsform (i.v. oder oral), des logistischen Aufwands (Durch- führung ambulant in der Arztpraxis nach Portimplantation versus zu Hause ohne vorbereitende Maßnahmen), der Anforderungen hinsichtlich Zuverlässigkeit und Selbstständigkeit (Risiko von Einnahmefehlern mit gravierenden Nebenwirkungen bei Einnahme mehrerer Tabletten täglich) und im möglichen Nebenwirkungsprofil ( $\bullet$ Tab. 1).

Herr K. und seine Frau entscheiden sich in Übereinstimmung mit dem behandelnden Onkologen für das etwas mildere und möglicherweise minimal weniger wirksame oral einzunehmende Chemotherapeutikum. Für diese Entscheidung spielt eine Rolle, dass Herr K. und seine Frau ihren Alltag nicht zu sehr durch die Chemotherapie beeinflusst wissen wollen, Herr K. nicht allzu viel Zeit in der Arztpraxis verbringen möchte, sondern sei- 
Tab. 1 Überblick über die möglichen Chemotherapieschemata im Vergleich [33].

\begin{tabular}{|c|c|c|c|}
\hline & Folfox4 & 5-FU/LV & $\begin{array}{l}\text { Capecitabine } \\
\text { (Handelsname: Xeloda }{ }^{\circledR} \text { ) }\end{array}$ \\
\hline Darreichungsform & \multicolumn{2}{|c|}{$\begin{array}{l}\text { über eine Vene (intravenös) über einen Port, } \\
\text { zur Anlage des Ports Operation erforderlich }\end{array}$} & $\begin{array}{l}\text { oral-durch den Mund } \\
\text { (Filmtabletten) }\end{array}$ \\
\hline Ablauf & \multicolumn{2}{|c|}{$\begin{array}{l}\text { - ambulant in der Arztpraxis } \\
\text { - } 12 \text { Behandlungen (Zyklen) über } 2 \text { Tage } \\
\text { alle } 2 \text { Wochen für } 6 \text { Monate } \\
\text { - ca. } 30 \text { Arztbesuche }\end{array}$} & $\begin{array}{l}\text { - zu Hause durchführbar (Ge- } \\
\text { fahr von Einnahmefehlern, da } \\
\text { täglich viele Tabletten einge- } \\
\text { nommen werden müssen!) } \\
\text { - zweimal täglich für } 14 \text { Tage, } \\
\text { 1 Woche Pause für } 6 \text { Monate } \\
\text { - ca. } 8 \text { Arztbesuche }\end{array}$ \\
\hline \multirow[t]{2}{*}{$\begin{array}{l}\text { Mögliche } \\
\text { Nebenwirkungen }{ }^{1}\end{array}$} & \multicolumn{3}{|c|}{$\begin{array}{l}\text { In unterschiedlicher Stärke und in Abhängigkeit vom allgemeinen Gesundheitszustand } \\
\text { und Alter: Übelkeit, Erbrechen, Appetitlosigkeit, Durchfall, Bauchschmerzen, Rücken- } \\
\text { schmerzen, Müdigkeit, Abgeschlagenheit, Entzündung der Mundschleimhaut, Blutarmut, } \\
\text { erhöhte Infektionsanfälligkeit, Blutungsneigung, vorübergehender leichter Haarausfall }\end{array}$} \\
\hline & $\begin{array}{l}\text { Nervenschäden (Neuropa- } \\
\text { thien): Gefühlsstörungen } \\
\text { wie Taubheit, Kribbeln, Schmer- } \\
\text { zen der Haut, Probleme mit dem } \\
\text { Tast- und Berührungsempfinden. } \\
\text { Bei den meisten Patienten vor- } \\
\text { übergehend, bei wenigen Patien- } \\
\text { ten auch dauerhaft }\end{array}$ & & $\begin{array}{l}\text { Hand-Fuß-Syndrom: schmerz- } \\
\text { hafte Schwellung von Händen } \\
\text { und Füßen/entzündliche Rö- } \\
\text { tung der Hand- und Fußflächen }\end{array}$ \\
\hline \multirow[t]{2}{*}{ Wirksamkeit $^{2}$} & $\begin{array}{l}\text { - vermutlich etwas wirksamer } \\
\text { - bei älteren Patienten (über } \\
\text { 70J.) nicht zu empfehlen } \\
\text { (Nebenwirkungen) }\end{array}$ & \multicolumn{2}{|c|}{ 5-FU/LV und Capecitabine vermutlich gleich wirksam } \\
\hline & $\begin{array}{l}{ }^{2} \text { nebenwirkungsreicher, } \\
\text { für jüngere Patienten geeignet }\end{array}$ & $\begin{array}{l}{ }^{2} \text { gute } \\
\text { Verträgl }\end{array}$ & $\begin{array}{l}{ }^{2} \text { gute } \\
\text { Verträglichkeit }\end{array}$ \\
\hline
\end{tabular}

${ }^{1}$ Wenn andere Erkrankungen vorliegen, fallen Nebenwirkungen möglicherweise gravierender aus. Die Liste der Nebenwirkungen ist nicht vollständig. In seltenen Fällen können noch andere Nebenwirkungen auftreten.

${ }^{2}$ Alle drei Protokolle haben eine gute Wirksamkeit. Folfox ist vermutlich etwas wirksamer. Bislang erlaubt die Studienlage allerdings keine sichere Aussage.

ne Zeit lieber der Familie und seinen Hobbys widmet. Außerdem traut er sich eine selbstständige und zuverlässige Einnahme der erforderlichen Medikation zu.

Herr K. ist zuversichtlich, eine gute und informierte Entscheidung getroffen $\mathrm{zu}$ haben, die er umsetzen kann und die zu seinen Bedürfnissen und Wertvorstellungen passt. Er ist sich im Klaren darüber, mit welchen unerwünschten Wirkungen er durch die orale Chemotherapie rechnen muss, Dies nimmt er seiner Familie zuliebe und bei großem Sicherheitsbedürfnis jedoch in Kauf.

\section{Die Wurzeln der PEF}

\section{$\nabla$}

Wichtige Impulse zur Entwicklung der PEF sind zum einen in der „Consumer-Rights“-Bewegung (Verbraucherschutz) zu sehen, die in den 60erund 70er-Jahren in Nordamerika ihren Ursprung nahm. Eine Grundannahme ist, dass Verbraucher den Anbietern von Dienstleistungen und Produkten (und im speziellen Fall Patienten ihren Behandlern) durch ein Informationsdefizit systembedingt unterlegen sind und daher eines besonderen Schutzes und besonderer Unterstützung bedürfen. Zum anderen war Ende der 90er-Jahre in den USA durch das umfassende „Dartmouth Atlas of Healtcare Projekt“ (www.dartmouthatlas. org) (Analyse von Daten aus 3436 Krankenhäusern) offensichtlich geworden, dass Behandlungsentscheidungen sehr stark abhängig von der Region sind, in der ein Patient lebt, und von der dort vorherrschenden medizinischen Meinung, und nicht etwa durch klare medizinische Evidenz oder aber durch die Berücksichtigung von Patientenpräferenzen verstehbar werden [36]. So zeigte sich beispielsweise dank der Erhebung, dass in einigen Regionen der USA bei Brustkrebs in frühem Stadium alle Frauen eine Brustamputation erhielten, keine einzige Frau brusterhaltend operiert wurde, wobei in anderen Regionen bei ungefähr 50\% aller Frauen ein Brusterhalt möglich war. Auch für andere Erkrankungen gab es eine regional abhängige erhebliche Varianz in den Operationsraten. Diese Erkenntnis stärkte die Position derer, die forderten, dass bei mehreren gleichwertigen Therapieoptionen und bei präferenzsensitiven Entscheidungen die Patientenperspektive verstärkt Berücksichtigung finden müsse.

\section{Beginn der PEF-Initiativen in Deutschland $\nabla$}

In Deutschland setzte das zunehmende gesundheitspolitische Interesse an der Partizipativen Entscheidungsfindung sowie Forschungsaktivitäten in diesem Bereich erst mit einer zeitlichen

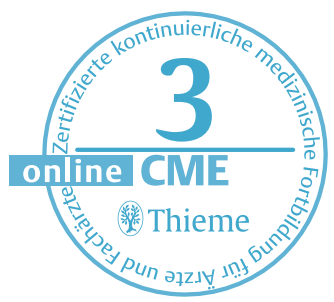


"Gesetz zur Verbesserung der Rechte von Patientinnen und Patienten“ (2013)

$\S 630$ e Aufklärungspflichten:

(1) Der Behandelnde ist verpflichtet,

- den Patienten über sämtliche für die Einwilligung wesentlichen Umstände aufzuklären.

- Dazu gehören insbesondere Art, Umfang, Durchführung, zu erwartende Folgen und Risiken der Maßnahme sowie ihre Notwendigkeit, Dringlichkeit, Eignung und Erfolgsaussichten im Hinblick auf die Diagnose oder die Therapie.

- Bei der Aufklärung ist auch auf Alternativen zur Maßnahme hinzuweisen, wenn mehrere medizinische gleichermaßen indizierte und übliche Methoden zu wesentlich

unterschiedlichen Belastungen, Risiken oder Heilungschancen führen können.

(2) Die Aufklärung muss

1. mündlich durch den Behandelnden oder durch eine Person erfolgen, die über die zur Durchführung der Maßnahme notwendige Ausbildung verfügt; ergänzend kann auch auf Unterlagen Bezug genommen werden, die der Patient in Textform erhält,

2. so rechtzeitig erfolgen, dass der Patient seine Entscheidung über die Einwilligung wohlüberlegt treffen kann,

3. für den Patienten verständlich sein.

Abb. 9

Auszug aus dem Patientenrechtegesetz von 2013

Verzögerung von ca. 10 Jahren ein. Als wichtiger Startschuss hierfür ist die Gründung des Förderschwerpunkts „Patient als Partner im Medizinischen Entscheidungsprozess“ durch das Bundesministerium für Gesundheit (BMG) im Jahr 2001 zu sehen. Damals lagen bereits einige internationale Studien vor, die günstige Behandlungseffekte der PEF erwarten ließen. Es war allerdings unklar, inwieweit sich dieses Behandlungsmodell auf das deutsche Gesundheitssystem übertragen lassen würde. In der deutschen Forschungslandschaft war das Thema PEF zu diesem Zeitpunkt noch weitgehend unbearbeitet. Ein erklärtes Ziel des BMG war es daher, durch die Förderung von Modellprojekten Wissen um die Effekte der PEF zu generieren und auch zu überprüfen, inwieweit sich internationale Forschungsergebnisse auf das deutsche Gesundheitswesen übertragen lassen. In einer ersten Förderphase des BMG-Förderschwerpunkts von 2001-2005 wurden 10 Modellprojekte sowie ein übergeordnetes Methodenprojekt gefördert, die die Effekte der PEF bei verschiedenen Erkrankungen erfassen sollten. Thematisch vertreten waren Erkrankungen aus dem psychoneurologischen Bereich (Depression, Schizophrenie, Alkoholismus, chronischer Schmerz, Therapiebegrenzung am Lebensende, Multiple Sklerose) sowie aus dem internistischchirurgischen Bereich (Brustkrebs, periphere arterielle Verschlusskrankheit, Hypertonie, Atemwegsinfekte bei Kindern). In den 10 Modellprojekten des BMG-Förderschwerpunkts ergaben sich durchweg günstige psychosoziale, klinische und auch entscheidungsprozessbezogene Effekte der PEF-Interventionen [36 - 44].

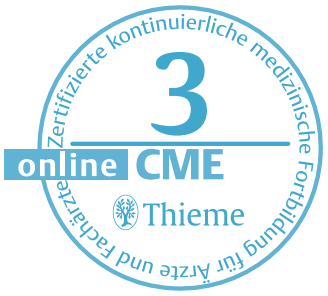

In einer 2. Förderphase dieses BMG-Förderschwerpunkts von 2005-2007 wurden daher im weiteren Verlauf 4 Forschungsprojekte gefördert, die erfolgreich Strategien zum Transfer der Partizipativen Entscheidungsfindung ins deutsche Gesundheitssystem entwickelten, implementierten und evaluierten (z.B. [23]).
Weitere große Forschungsinitiativen im Bereich Patientenbeteiligung folgten, beispielsweise unter Federführung des Bundesministeriums für Bildung und Forschung (BMBF) ab 2007 im Förderschwerpunkt zur versorgungsnahen Forschung „Chronische Krankheiten und Patientenorientierung" (www.forschung-patientenorientierung. de) sowie in einer zweiten Förderphase ab 2011.

\section{Gesundheitspolitischer Hintergrund der Patientenbeteiligung in Deutschland}

Es besteht mittlerweile ein Konsens im deutschen Gesundheitswesen, dass Patienten bei medizinischen Entscheidungen miteinbezogen werden sollten. Dies fand zunächst im Jahr 2007 seinen Ausdruck in einem gemeinsam von den Bundesministerien für Gesundheit und für Justiz herausgegebenen Leitfaden zu Patientenrechten in Deutschland [2]. Dieser klärt Patienten über Rechte und Pflichten im Arzt-Patienten-Verhältnis auf. Der Begriff PEF fällt im Leitfaden zwar nicht explizit, es werden aber eindeutig PEFMerkmale beschrieben. Das Dokument wurde gemeinsam von allen beteiligten Akteuren des Gesundheitswesens erarbeitet und setzte eine Landmarke in diesem Bereich. Ganz besonders wurde darin die Bedeutung des persönlichen Gesprächs zwischen Arzt und Patient hervorgehoben. Die Arzt-Patient-Beziehung sollte zukünftig als „echte Behandlungs- und Entscheidungspartnerschaft“" verstanden werden. In dem Leitfaden wurde auch festgeschrieben, welchen Anforderungen die Aufklärung über medizinische Behandlungen genügen muss:

- „Kommen mehrere gleichwertige medizinische Behandlungen oder Behandlungsmethoden in Betracht, muss der Arzt über Chancen und Risiken umfassend aufklären." (S.8) [2]

- "Zu unterrichten ist auch über Art und Wahrscheinlichkeit der verschiedenen Risiken im Verhältnis zu den Heilungschancen und über alternative Behandlungsmöglichkeiten.“ (S.11) [2]

- „Der Patient muss durch die Aufklärung in die Lage versetzt werden, beurteilen zu können, was die konkret vorgesehene Behandlung für ihn persönlich bedeuten kann." (S.11) [2]

Das Dokument ebnet somit den Weg hin zu einer größeren Patientenorientierung und einer partnerschaftlichen Beteiligung von Patienten im deutschen Gesundheitswesen und mündete im Jahr 2013 in einem Patientenrechtegesetz, das u. a. für eine Stärkung der Patientenbeteiligung und der Patienteninformation sorgt ( $\bullet$ Abb.9).

Allerdings muss erwähnt werden, dass das Patientenrechtegesetz in seinen Inhalten hinter den Ausführungen des Leitfadens zurückblieb und im Wesentlichen eine umfangreiche Aufklärung festschreibt sowie Regeln für den Fall eines medizinischen Behandlungsfehlers enthält. 
Bemerkbar macht sich eine größere Patientenorientierung auch durch strukturelle Maßnahmen auf unterschiedlichen Ebenen [45]. So vertritt z. B. seit dem Jahr 2004 ein Patientenbeauftragter des Bundes die Belange der Patienten in allen relevanten gesellschaftlichen Bereichen und soll die Weiterentwicklung der Patientenrechte unterstützen. Inwieweit dies tatsächlich zu einer größeren Patientenorientierung führt, muss jedoch erst noch belegt werden. Im Gemeinsamen Bundesausschuss haben Patientenvertreter Vorschlagsund Beratungsrechte. Verschiedene nationale Institutionen wurden gegründet, die die medizinischen Entscheidungen von Patienten im weiteren Sinne unterstützen, so z. B. das Institut für Qualität und Wirtschaftlichkeit im Gesundheitswesen (IQWIG), das Ärztliche Zentrum für Qualität in der Medizin (ÄZQ), Patientenuniversitäten in Hannover und Jena.

\section{Ausblick}

$\nabla$

Die Vorteile der PEF sind offensichtlich und Aktivitäten zur Implementierung der PEF im deutschen Gesundheitswesen nehmen zu. Bis PEF sich in der Regelversorgung durchsetzen wird, ist es allerdings noch ein weiter Weg. Neben einer Offenheit der Behandler für das PEF-Konzept im Sinne einer patientenzentrierten Herangehensweise, sind spezielle kommunikative Fertigkeiten eine wichtige Voraussetzung für die Umsetzung der PEF und sollten in der Aus- und Weiterbildung zunehmend Beachtung finden. Im Medizinstudium wird PEF-Kommunikation mittlerweile an vielen Ausbildungsstätten unterrichtet [46], von den Studierenden günstig aufgenommen und oft überraschend schnell umgesetzt. In der Weiterbildung von bereits langjährig praktizierenden Medizinern erweist es sich als größere Herausforderung, über Jahre eingeübte Kommunikationsstrategien weg von der traditionell üblichen paternalistischen Haltung hin zu mehr Partizipation zu erreichen $[23,26]$. Neue Strategien setzen, statt wie bisher auf PEF-Gruppenschulungen von Ärzten, nun auch auf PEF-Einzelcoachingansätze sowie auf e-Tutorials zur PEF, die von den Ärzten ort- und zeitunabhängig absolviert werden können (www.pefmed.de).

Interessenkonflikt: Die Erstautorin hat Fördermittel vom Bundesministerium für Gesundheit (BMG) und von der Deutschen Krebshilfe (DKH) zu mehreren wissenschaftlichen Forschungsprojekten zur Partizipativen Entscheidungsfindung erhalten.

\section{Fazit für die Praxis}

Das Konzept der PEF gilt mittlerweile als die ideale Form der Arzt-Patient-Interaktion, empfiehlt sich aus ethisch-juristischer Sicht und führt zu günstigen Effekten bei Patienten und Behandlern. Ursprünglich aus dem angloamerikanischen Raum kommend, setzt es sich in den letzten 15 Jahren zunehmend auch im deutschen Gesundheitssystem durch, da es von der Gesundheitspolitik gefördert und von Patienten begrüßt und gewünscht wird. Es empfiehlt sich insbesondere beim Vorliegen mehrerer gleichwertiger Behandlungsoptionen und bei präferenzsensitiven Entscheidungen. Eine wichtige Anwendungsvoraussetzung ist die patientenzentrierte Haltung des Behandlers. Konkrete Umsetzungsschritte, einschließlich Risikokommunikationsstrategien, können in PEF-Trainings erlernt werden. Der Einsatz von spezifischen Entscheidungshilfematerialien kann die Anwendung von PEF erleichtern, indem Entscheidungsgespräche vorbereitet, ergänzt, strukturiert und zeitlich entlastet werden.

\section{Literatur}

1 Härter $M$. Shared decision making - from the point of view of patients, physicians and health politics is set in place. Z Arztl Fortbild Qualitatssich 2004; 98: 89-92

2 Bundesministerium für Gesundheit. Patientenrechte in Deutschland. Leitfaden für Patientinnen/Patienten und Ärztinnen/Ärzte. 5. Aufl. Berlin: 2007

3 Elwyn G, Frosch $D$, Thomson $R$ et al. Shared decision making: a model for clinical practice. J Gen Intern Med 2012; 27: $1361-1367$

4 Charles C, Gafni A, Whelan T. Decision-making in the physician-patient encounter: revisiting the shared treatment decision-making model. Soc Sci Med 1999; 49: $651-661$

5 Charles C, Gafni A, Whelan T. Shared decision-making in the medical encounter: what does it mean? (or it takes at least two to tango) Soc Sci Med 1997; 44: 681-692

6 Towle A, Godolphin W. Framework for teaching and learning informed shared decision making. BMJ 1999; 319: $766-771$

7 Elwyn G, Edwards A, Kinnersley P. Shared decision-making in primary care: the neglected second half of the consultation. Br J Gen Pract 1999; 49: 477 - 482

8 Ernst J, Schwarz R, Krauß O. Shared decision making bei Tumorpatienten. Ergebnisse einer empirischen Studie. J Public Health 2004; 12: 123-131

9 Charles C, Whelan T, Gafni A. What do we mean by partnership in making decisions about treatment? BM] 1999; 319: $780-782$

10 Böcken J, Braun B, Schnee M, Hrsg. Gesundheitsmonitor 2004. Die ambulante Versorgung aus Sicht von Bevölkerung und Ärzteschaft. 1. Aufl. Gütersloh: Verlag Bertelsmann Stiftung; 2004

11 Böcken J, Braun B, Meierjürgen R, Hrsg. Gesundheitsmonitor 2014, Bürgerorientierung im Gesundheitswesen. 1. Aufl. Gütersloh: Bertelsmann Stiftung, Barmer GEK; 2014

12 Coulter A, Magee H. The European Patient of the Future State of Health. Maidenhead: Open University Press, McGraw Hill Companies; 2003

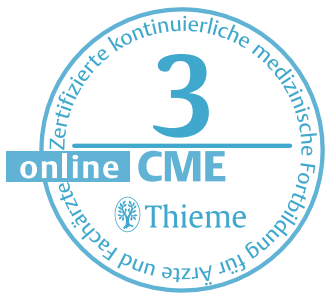


13 Klemperer D, Rosenwirt M. Shared Decision Making: Konzept, Voraussetzungen und politische Implikationen. Chartbook. Gütersloh: Bertelsmann Stiftung, Universität Bremen; 2005

14 Floer B, Schnee M, Bocken J et al. Shared decision making. The perspective of practicing physicians. Med Klin (Munich) 2004; 99: 435-440

15 Legare F, Ratte S, Gravel $K$ et al. Barriers and facilitators to implementing shared decision-making in clinical practice: update of a systematic review of health professionals' perceptions. Patient Educ Couns 2008; 73: 526 535

16 Legare $F$, Thompson-Leduc $P$. Twelve myths about shared decision making. Patient Educ Couns 2014; 96: 281 - 286

17 Joseph-Williams N, Elwyn G, Edwards A. Knowledge is not power for patients: A systematic review and thematic synthesis of patient-reported barriers and facilitators to shared decision making. Patient Educ couns 2014; 94: $291-309$

18 Elwyn G, Edwards A, Kinnersley P et al. Shared decision making and the concept of equipoise: the competences of involving patients in healthcare choices. Br J Gen Pract 2000; 50: 892-899

19 Whitney SN. A new model of medical decisions: exploring the limits of shared decision making. Med Decis Making 2003; 23: 275-280

20 Kiesler DJ, Auerbach SM. Optimal matches of patient preferences for information, decision-making and interpersonal behavior: evidence, models and interventions. Patient Educ Couns 2006; 61: 319-341

21 Elwyn G, Tsulukidze M, Edwards A et al. Using a 'talk' model of shared decision making to propose an observation-based measure: Observer OPTION 5 Item. Patient Educ Couns 2013; 93: 265-271

22 Loh A, Simon D, Rockenbauch $K$ et al. Shared Decision Making - importance and dissemination in medical education. Z Med Psychol 2006; 15: 87-92

23 Bieber C, Nicolai J, Hartmann $M$ et al. Training physicians in shared decisionmaking - who can be reached and what is achieved? Patient Educ Couns 2009; 77: $48-54$

24 Bieber CLA, Ringel N, Eich W, Härter M. Patientenbeteiligung bei medizinischen Entscheidungen: Manual zur Partizipativen Entscheidungsfindung (Shared Decision Making). Heidelberg: Universitätsklinikum Heidelberg; 2007

25 Elwyn G, Edwards A, Hood K et al. Achieving involvement: process outcomes from a cluster randomized trial of shared decision making skill development and use of risk communication aids in general practice. Fam Pract 2004; 21: $337-346$

26 Härter M, Buchholz A, Nicolai J et al. Shared Decision Making and the Use of Decision Aids. Dtsch Arztebl Int 2015; 112: 672 -679

27 Gurm HS, Litaker DG. Framing procedural risks to patients: is $99 \%$ safe the same as a risk of 1 in 100? Acad Med 2000; 75: 840-842

28 Hamann J, Mendel R, Meier A et al. "How to speak to your psychiatrist": shared decision-making training for inpatients with schizophrenia. Psychiatr Serv 2011; 62: 1218-1221

29 Stacey $D$, Bennett CL, Barry MJ et al. Decision aids for people facing health treatment or screening decisions. Cochrane Database Syst Rev 2011: CD001431

30 Elwyn G, O'Connor A, Stacey D et al. Developing a quality criteria framework for patient decision aids: online international Delphi consensus process. BM] 2006; 333: 417
31 Caspari C, Vodermaier A. Entscheidungshilfe für Frauen mit Brustkrebs: Brusterhaltende Therapie oder Brustentfernung? Gemeinsame Planung der Operation bei kleinem Tumor. Berlin: AOK Bundesverband; 2008

32 Loh A, Simon D, Kriston L et al. Patientenbeteiligung bei medizinischen Entscheidungen. Effekte der Partizipativen Entscheidungsfindung aus systematischen Reviews. Dtsch Arztebl 2007; 104: A1483-A1488

33 Stacey $D$, Legare $F, C o l N F$ et al. Decision aids for people facing health treatment or screening decisions. Cochrane Database Syst Rev 2014; 1: CD001431

34 Bieber C, Nicolai J. Behandlung nach der Operation beim Dickdarmkrebs (Kolonkarzinom) im Stadium II: Unterstützende (adjuvante) Chemotherapie - Ja oder Nein? Entscheidungshilfe zur Gemeinsamen Planung der weiteren Behandlungsschritte. 1. Aufl. Heidelberg: Universitätsklinikum Heidelberg; 2010

35 Wennberg JE. Unwarranted variations in healthcare delivery: implications for academic medical centres. BM] 2002; 325: 961 - 964

36 Hochlehnert A, Richter A, Bludau HB et al. A computer-based informationtool for chronic pain patients. Computerized information to support the process of shared decision-making. Patient Educ Couns 2006; 61: 92 - 98

37 Bieber C, Muller KG, Blumenstiel $K$ et al. Long-term effects of a shared decision-making intervention on physician-patient interaction and outcome in fibromyalgia. A qualitative and quantitative 1 year follow-up of a randomized controlled trial. Patient Educ Couns 2006; 63: 357 - 366

38 Loh A, Simon D, Wills CE et al. The effects of a shared decision-making intervention in primary care of depression: a cluster-randomized controlled trial. Patient Educ Couns 2007; 67: 324-332

39 Loh A, Leonhart $R$, Wills CE et al. The impact of patient participation on adherence and clinical outcome in primary care of depression. Patient Educ Couns 2007; 65: 69-78

40 Vodermaier A, Caspari C, Koehm J et al. Contextual factors in shared decision making: a randomised controlled trial in women with a strong suspicion of breast cancer. Br J Cancer 2009; 100: 590 - 597

41 Vodermaier $A$, Caspari $C$, Wang $L$ et al. How and for whom are decision aids effective? Long-term psychological outcome of a randomized controlled trial in women with newly diagnosed breast cancer. Health Psychol 2011; 30: $12-19$

42 Hamann J, Cohen R, Leucht S et al. Do patients with schizophrenia wish to be involved in decisions about their medical treatment? Am J Psychiatry 2005; 162: $2382-2384$

43 Hamann J, Cohen R, Leucht $S$ et al. Shared decision making and long-term outcome in schizophrenia treatment. J Clin Psychiatry 2007; 68: 992 - 997

44 Hamann J, Langer B, Winkler $V$ et al. Shared decision making for in-patients with schizophrenia. Acta Psychiatr Scand 2006; 114: 265 - 273

45 Härter M, Muller H, Dirmaier / et al. Patient participation and shared decision making in Germany - history, agents and current transfer to practice. Z Evid Fortbild Qual Gesundhwes 2011; 105: 263 - 270

46 Loh A, Simon D, Rockenbauch $K$ et al. Partizipative Entscheidungsfindung Stellenwert und Verbreitung in der medizinischen Ausbildung. Z Med Psychol 2006; 15: 87-92

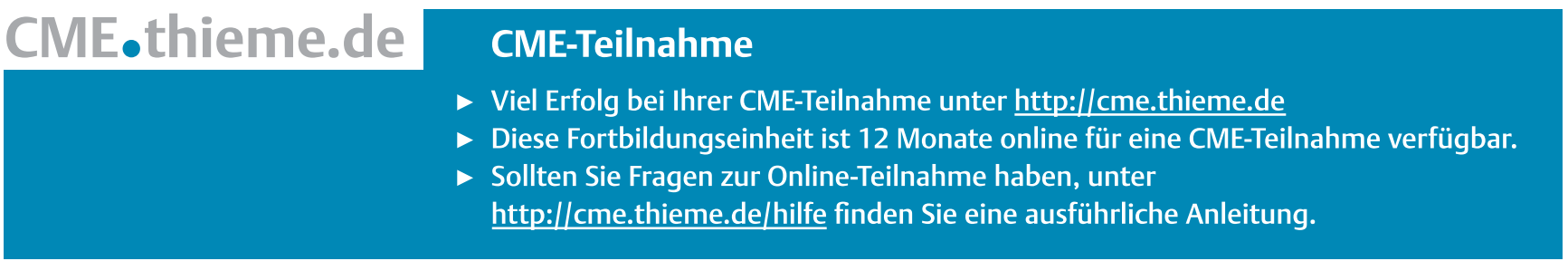




\section{CME-Fragen Partizipative Entscheidungsfindung (PEF) - Patient und Arzt als Team}

1 Welche Antwort ist richtig? Die Autonomie des Patienten ist am größten beim Vorgehen nach... dem Deliberativen Modell. dem Paternalistischen Modell. dem Informed Consent Modell. dem Informationsmodell. dem Modell der Partizipativen Entscheidungsfindung.

2 Welche Antwort ist falsch? Die Partizipative Entscheidungsfindung ist besonders dann geeignet, wenn...

A mehrere gleichwertige Behandlungsoptionen zur Verfügung stehen.

B eine Notfallsituation vorliegt.

C der Patient eine aktive Beteiligung wünscht.

D sich mögliche Behandlungsoptionen im Risiko-NutzenProfil deutlich unterscheiden.

E der Arzt die Verantwortung nicht alleine übernehmen kann und will.

3 Welche Antwort ist falsch? Zu den 6 Prozessschritten der Partizipativen Entscheidungsfindung gehört...

A der Einsatz von schriftlichen Informationsmaterialien. das Erfragen der Rollenpräferenz des Patienten. die Problemdefinition.

D das Erfragen von Erwartungen und Befürchtungen des Patienten.

E das Beschreiben der Behandlungsoptionen.

4 Welche Antwort ist falsch? Der Einsatz von Partizipativer Entscheidungsfindung führt beim Patienten zu ... geringeren Entscheidungskonflikten. einer aktiveren Beteiligung. einer schnelleren Genesung. einer besseren Therapietreue.

E realistischeren Erwartungen über Behandlungsverläufe.

Welche Antwort ist richtig? Im Arzt-Patient-Kontakt wird die Umsetzung von PEF erleichtert durch... den Einsatz von Entscheidungshilfen. einen gewissen Zeitdruck. kognitive Einschränkungen des Patienten. eine paternalistische Haltung des Arztes. die vorherige Internetrecherche des Patienten.

6 Welche Antwort ist falsch? Deutschsprachige Entscheidungshilfen liegen vor für... die Behandlung bei Brustkrebs. das Screening auf ein Prostatakarzinom. die Behandlung bei Darmkrebs. die Behandlung der Depression.

E die Behandlung der Anorexie.
7 Welche Antwort ist richtig? Partizipative Entscheidungsfindung sollte besonders dann stattfinden, wenn...

A die Bedeutsamkeit für den Patienten sehr hoch ist und die medizinische Evidenz gering.

B die medizinische Evidenz sehr hoch ist und die Bedeutsamkeit für den Patienten gering.

C die medizinische Evidenz mäßig ist und die Bedeutsamkeit für den Patienten hoch.

D die Bedeutsamkeit für den Arzt sehr hoch ist und die medizinische Evidenz gering.

E die medizinische Evidenz sehr hoch ist und die Bedeutsamkeit für den Arzt sehr hoch.

8 Welche Antwort ist richtig? Bei der Partizipativen Entscheidungsfindung...

A entscheidet der Arzt, nachdem er sich die Wünsche seines Patienten angehört hat.

B entscheidet der Patient, nachdem der sich die medizinischen Informationen seines Arztes angehört hat.

C entscheiden Arzt und Patient gemeinsam, nachdem sich der Patient selbstständig im Internet informiert hat.

D entscheiden Arzt und Patient gemeinsam, ohne sich gegenseitig zu beeinflussen.

E entscheiden Arzt und Patient gemeinsam, nachdem sie sich über persönliche und medizinische Informationen ausgetauscht haben.

9 Welche Antwort ist falsch? Für die Partizipative Entscheidungsfindung gilt:

A Sie wird von der Mehrzahl der Ärzte umgesetzt.

B Sie wird von der Mehrzahl der gesunden Bürger favorisiert.

C Sie wird von der Mehrzahl der Ärzte favorisiert.

D Sie wird von der Mehrzahl der Patienten gewünscht.

E Sie wird von der Minderheit der Ärzte abgelehnt.

Welche Antwort ist richtig? Patienten fühlen sich...

A häufiger zu Fragen aufgefordert, als ihre Ärzte glauben.

B schlechter zu Vor- und Nachteilen einer Behandlung informiert, als ihre Ärzte denken.

C häufiger zur Nutzung anderer Informationsquellen ermuntert, als ihre Ärzte denken.

D häufiger mit ihren Vorstellungen einbezogen, als ihre Ärzte denken.

E schlechter zu Behandlungsalternativen informiert, als ihre Angehörigen denken. 